\title{
A functional assessment of the circle of Willis before aortic arch surgery using transcranial Doppler
}

\author{
Tim Smith, MD, PhD, ${ }^{a}$ Giuliano Jafrancesco, MD, ${ }^{a}$ Giusy Surace, MD, ${ }^{a}$ Wim J. Morshuis, MD, PhD, ${ }^{b}$ \\ Selma C. Tromp, MD, PhD, ${ }^{\mathrm{c}}$ and Robin H. Heijmen, $\mathrm{MD}, \mathrm{PhD}^{\mathrm{a}, \mathrm{d}}$
}

\section{ABSTRACT}

Background: Antegrade selective cerebral perfusion (ASCP) with systemic moderate hypothermia is routinely used as brain protection during aortic arch surgery. Whether ASCP should be delivered unilaterally (u-ASCP) or bilaterally (biASCP) remains controversial.

Methods: We routinely studied the functional anatomy of the circle of Willis (CoW in all patients scheduled for arch surgery using transcranial color-coded Doppler over a decade. On the basis of these data, we classified observed functional variants as being "safe," "moderately safe," or "unsafe" for u-ASCP.

Results: From January 2005 to June 2015, 1119 patients underwent aortic arch surgery in our institution. Of these, 636 patients had elective surgery performed with ASCP. Preoperative full functional assessment of the $\mathrm{CoW}$ was possible in $61 \%$ of patients. A functionally complete CoW was found in only $27 \%$. Of all variants, $72 \%$ were classified as being safe for u-ASCP, whereas $18 \%$ were moderately safe for u-ASCP, and 10\% unsafe. Unsafe variants for bi-ASCP were observed in $0.5 \%$ of patients.

Conclusions: The risk of ischemic brain damage due to malperfusion is estimated to be substantially higher during right u-ASCP than during bi-ASCP. Bi-ASCP is therefore highly preferable over u-ASCP if the function of the CoW is unknown. We propose a tailored approach using this full functional assessment preoperatively by applying $\mathrm{u}$-ASCP via the right subclavian artery when considered safely possible, and bi-ASCP when considered a necessity to prevent cerebral malperfusion, and thus thereby try to reduce the embolic stroke risk of ostial instrumentation in bi-ASCP. (J Thorac Cardiovasc Surg 2019;158:1298-304)

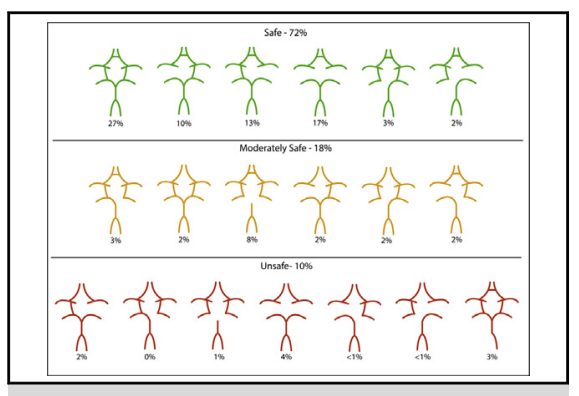

Functional variants of the circle of Willis, correlated to safety of u-ASCP.

\section{Central Message}

On the basis of our functional assessment of the circle of Willis of 636 arch surgery patients, unilateral ASCP is safely possible in $72 \%$ of patients. In $28 \%$, u-ASCP would lead to cerebral malperfusion.

\section{Perspective}

The benefit of u-ASCP is avoidance of ostial manipulation and thus theoretically lower embolic stroke risk; bi-ASCP is theoretically preferable over u-ASCP in patients with incomplete CoW because of the risk of malperfusion. Although anatomical studies have been performed, no literature is available on the functional anatomy of the $\mathrm{CoW}$ in patients scheduled for aortic arch surgery.

See Commentary on page 1305 .
Antegrade selective cerebral perfusion (ASCP) with systemic moderate hypothermia has become the routine technique for brain protection during aortic arch surgery. A recent

\footnotetext{
From the Departments of ${ }^{\mathrm{a} C a r d i o t h o r a c i c ~ S u r g e r y, ~ a n d ~}{ }^{\mathrm{c} C l i n i c a l}$ Neurophysiology, St Antonius Hospital, Nieuwegein, The Netherlands; ${ }^{b}$ Department of Cardiothoracic Surgery, University Medical Center St Radboud, Nijmegen, The Netherlands; and ${ }^{\mathrm{d}}$ Department of Cardiothoracic Surgery, Academic Medical Center Amsterdam, Amsterdam, The Netherlands.

Drs Smith and Jafrancesco contributed equally to this work.

Received for publication April 13, 2018; revisions received Oct 31, 2018; accepted for publication Jan 3, 2019; available ahead of print Feb 22, 2019.

Address for reprints: Robin H. Heijmen MD, PhD, Department of Cardiothoracic Surgery, St Antonius Hospital, Nieuwegein, The Netherlands (E-mail: r.heijmen@ antoniusziekenhuis.nl).

$0022-5223 / \$ 36.00$

Copyright (c) 2019 by The American Association for Thoracic Surgery

https://doi.org/10.1016/j.jtcvs.2019.01.007
}

European survey showed that approximately two-thirds of centers prefer bilateral ASCP (bi-ASCP), whereas onethird of them deliver the cerebral perfusate in an unilateral manner. ${ }^{1}$ Deep hypothermic circulatory arrest or retrograde cerebral perfusion is rarely used in Europe.

Unilateral ASCP (u-ASCP) is usually performed using a right subclavian-axillary approach, although some prefer

- Scanning this QR code will take you to the article title page to access supplementary information.

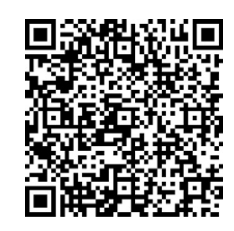




\section{Abbreviations and Acronyms \\ A1 $=$ anterior cerebral artery segment \\ $\mathrm{ACoA}=$ anterior communicating artery \\ ASCP = antegrade selective cerebral perfusion \\ bi-ASCP = bilateral antegrade selective cerebral perfusion \\ $\mathrm{CoW}=$ circle of Willis \\ $\mathrm{P} 1$ and $\mathrm{P} 2=$ posterior cerebral artery segment \\ $\mathrm{PCoA}=$ posterior communicating artery \\ PSV = peak systolic velocity \\ TCCD = transcranial color-coded duplex \\ $\mathrm{TCD}=$ transcranial Doppler \\ $\mathrm{u}$-ASCP $=$ unilateral antegrade selective cerebral perfusion}

direct cannulation of the brachiocephalic trunk or even the left common carotid artery. ${ }^{1} \mathrm{U}-\mathrm{ASCP}$, however, relies on adequate collaterals from right-to-left and/or from posterior-to-anterior for brain perfusion. Apart from the variable existence of extracranial and leptomeningeal connections, collateral flow in the brain (including both hemispheres, the brain stem, and cerebellum) is mainly dependent on the circle of Willis (CoW). The CoW, however, is known to be incomplete in more than half of the population, risking suboptimal brain protection during u-ASCP despite its reduced metabolism at moderate hypothermia.

Previously, Papantchev and colleagues ${ }^{2}$ and Merkkola and colleagues ${ }^{3}$ have studied the existing CoW variations in the general population, and their potential detrimental effect on global cerebral perfusion when applying u-ASCP, using an anatomical dissection study and imaging and casting study in human cadavers, respectively. Cerebral collateral circulation, however, depends on a more dynamic collateral capacity than strictly anatomical.

For more than a decade, we have routinely used transcranial Doppler (TCD) for intraoperative cerebral flow monitoring during ASCP, aside bilateral radial artery pressure monitoring, electroencephalography, and near infrared spectroscopy. ${ }^{4}$ As part of the preoperative workup, all of our elective patients are studied extensively for existing variations or pathological findings in cerebropetal vasculature and CoW using extracranial and transcranial color-coded duplex (TCCD) sonography. Nonetheless, we routinely use bi-ASCP in most of our patients. In our strive to further reduce operative stroke rate, these available data in more than hundreds of aortic arch patients, providing a true functional analysis of the CoW, prompted us to retrospectively analyze existing variations in collateral capacity and their potential effect on brain protection when using u-ASCP instead of bi-ASCP as a routine. Using u-ASCP when collateral capacity is considered safe, and bi-ASCP only when considered a necessity might reduce surgical manipulation at the ostia of the cerebral vessels, and hence potentially reduce embolic stroke rate.

\section{METHODS}

\section{Study Design and Population}

We performed a single-center, retrospective observational study. From January 2005 to June 2015, 1119 patients underwent aortic arch surgery in our institution. ASCP was used in 1033 cases (92\%), and deep hypothermic circulatory arrest in 86 cases $(8 \%)$. After excluding all nonelective ASCP cases, a total of 636 patients were studied, being accepted for elective aortic arch surgery, and hence had undergone duplex ultrasonography of the carotid and vertebral arteries, and TCCD measurements as part of our routine preoperative workup.

\section{Functional Evaluation of the CoW}

Ultrasound assessment of the bilateral common, internal, and external carotid artery, and both vertebral arteries (L9-3 transducer, iU22 ultrasound system, Philips, Eindhoven, The Netherlands), together with TCCD measurements (S5-1 transducer, iU22 ultrasound system, Philips) were performed as part of the routine preoperative workup. If the middle part of the common carotid artery was free of atherosclerotic disease or dissection, carotid compression was performed during a few heartbeats to assess the collateral function of the CoW. Carotid compression was considered effective when leading to a dampened wave signal of the plethysmogram measured on the ipsilateral earlobe. The vertebral artery was either normal, occluded, or classified as hypoplastic when absolute diameter was $<2 \mathrm{~mm}$, or $<50 \%$ of the contralateral vertebral artery diameter. 5

The middle, anterior (A1), and posterior (P1 and P2) cerebral arteries were insonated through the ipsilateral or, if necessary, contralateral temporal bone window. The vertebral and basilar arteries were insonated through the foramen magnum. The collateral capacity of the posterior communicating artery ( $\mathrm{PCOA}$ ) was assessed by measuring the peak systolic velocity (PSV) in the posterior cerebral artery (P1) during ipsilateral carotid compression. Subsequently, the collateral capacity of the anterior communicating artery (ACoA0 was assessed by measuring the PSV in A1 during contralateral carotid compression. When the PSV increased $>20 \%$, the collateral capacity of the vessel was considered normal. ${ }^{6}$ When the PSV increased $<20 \%$, the collateral capacity was considered moderate; when the PSV did not change, the communication was considered absent. We classified the collateral capacity of a communicating artery as "dysfunctional" when the function was moderate or absent (ie PSV increase $<20 \%$ ). The absence of P1 was classified as "fetal variant" (ie, posterior cerebral artery perfused by the ipsilateral internal carotid artery via $\mathrm{PCoA}$ ).

\section{Classification of Functional Variants}

During right-sided u-ASCP, the right internal carotid artery and right vertebral artery are perfused directly and perfusion of the left hemisphere might be hampered. We assumed that collateral perfusion would be adequate, and thus right u-ASCP would be safe, as long as the collateral flow did not have to reach a vessel beyond the left middle cerebral artery. This principle is illustrated in Figure 1. An example of a safe functional variant is an isolated dysfunction of the left PCoA. In this variant, perfusion of the left A1 and left middle cerebral artery is possible through the ACoA, and perfusion of the left posterior cerebral artery is possible via the right vertebral artery and basilar artery. Note that collateral flow does not reach a vessel beyond the left middle cerebral artery.

If cerebral territories were isolated from the CoW-dependent collateral perfusion during right $\mathrm{u}-\mathrm{ASCP}$, the functional variant was classified as unsafe. Right u-ASCP was considered moderately safe if right-to-left of posterior-to-anterior collateral perfusion had to reach a vessel beyond the left middle artery. For instance, in a left fetal variant (aplasia of the left 


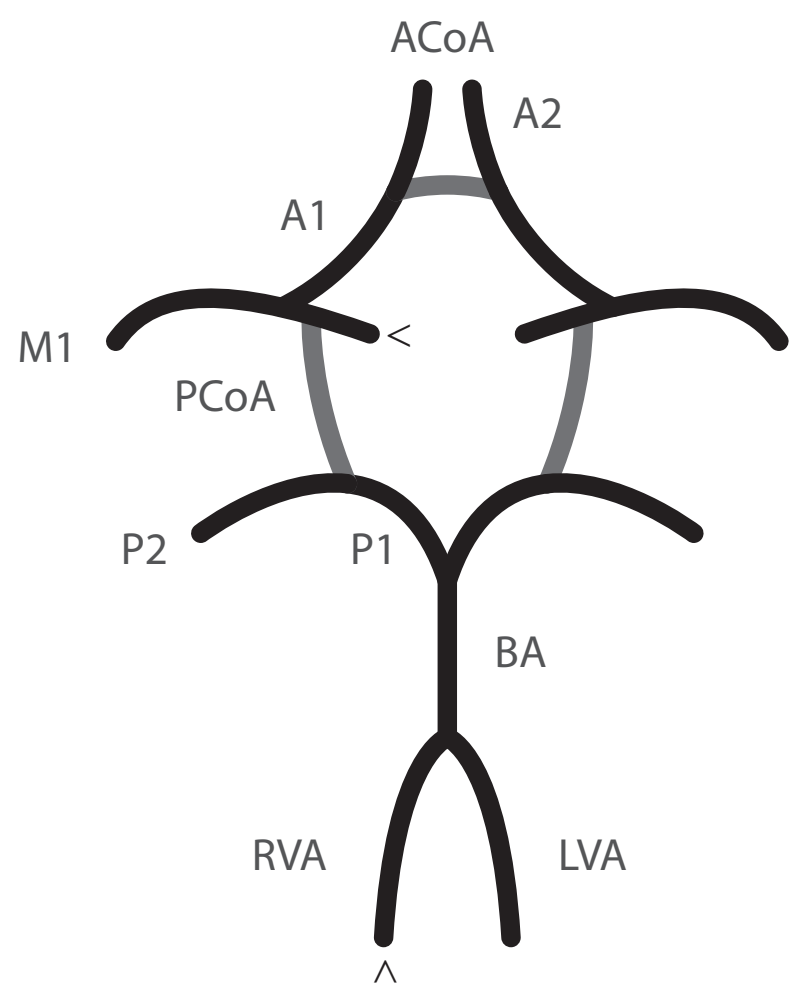

FIGURE 1. Schematic representation of the circle of Willis during u-ASCP. The arrow indicates blood supply to the right internal carotid artery and right vertebral artery during right-sided u-ASCP via the right subclavian artery. $A C o A$, Anterior communicating artery; $A 2$, anterior cerebral artery segment $2 ; A 1$, anterior cerebral artery segment $1 ; M 1$, middle cerebral artery segment $1 ; P C o A$, posterior communicating artery; $P 2$, posterior cerebral artery segment $2 ; P 1$, posterior cerebral artery segment $1 ; B A$, basilar artery; $R V A$, right vertebral artery; $L V A$, left vertebral artery.

P1 segment) collateral flow to the left posterior cerebral artery (P2) has to come from the right carotid artery, via right $\mathrm{A} 1, \mathrm{ACoA}$, left $\mathrm{A} 1$, and left $\mathrm{PCoA}$, thus reaching downstream to the left middle cerebral artery. BiASCP was considered unsafe when the right vertebral artery would be dysfunctional, combined with either bilateral PCoA dysfunction or combined with a fetal variant. Both of these variants would leave the posterior territory isolated from CoW-dependent collateral flow. On the basis of these assumptions, we proposed a clinical "traffic light" algorithm classifying different functional anatomical variants as safe (green light), as moderately safe (amber), and as unsafe (red light) for u-ASCP.

\section{Operative Technique}

All patients were operated using a midsternal approach. After heparinization, cardiopulmonary bypass was instituted with an arterial cannula either in the ascending aorta, or in the common femoral artery, and a venous 2 -staged canula in the right atrium in most patients. Patients were cooled to nasopharyngeal and core temperature of $25^{\circ} \mathrm{C}$, followed by systemic arrest and opening of the aorta. With the patient in the Trendelenburg position, two 15-French retrograde coronary sinus perfusion cannulas (DLP, Medtronic, Inc, Minneapolis, Minn) connected to the oxygenator with a separate pump head (single roller or centrifugal) were inserted into the ostia of the innominate and left common carotid arteries. The left subclavian artery was either externally clamped or occluded with a balloon catheter to avoid the steal phenomenon through the ipsilateral vertebral artery. Cerebral perfusion was initiated at a rate of $10 \mathrm{~mL} / \mathrm{min} / \mathrm{kg}$ and adjusted to maintain a right radial artery pressure between 40 and $70 \mathrm{~mm} \mathrm{Hg}$. All details of our operative technique are also described in our report by Cefarelli and colleagues. ${ }^{16}$

\section{RESULTS}

We studied 636 patients who underwent elective aortic arch surgery with ASCP, and hence had undergone duplex ultrasonography of the carotid and vertebral arteries, and TCCD measurements as part of our routine preoperative workup. Of these patients, $132(21 \%)$ had insufficient temporal bone windows to assess all intracranial large vessels, $95(15 \%)$ did not tolerate common carotid artery compression because of enhanced cardio-inhibitory response, and $18(3 \%)$ had carotid disease precluding carotid compression. The final study population consisted of 391 patients $(61 \%)$ who had complete functional evaluation of the CoW using TCCD. The mean age of the patients was $62 \pm 12$ years, and $61 \%$ were male $(n=237)$.

\section{Functional Anatomy of the CoW}

The prevalence of all functional variants is described in Table 1. A functionally complete $\mathrm{CoW}$ was found in only $27 \%$ of patients $(n=107)$. The most common functional variants were dysfunction of the left and right PCoAs $(17 \% ; n=65)$, dysfunction of the left PCoA $(13 \%$; $\mathrm{n}=50)$, and dysfunction of the right PCoA $(10 \% ; \mathrm{n}=41)$.

\section{Classification of Functional Variants}

As discussed in the Methods section, we classified the functional variants as safe (green light), as moderately safe (amber), or as unsafe (red light) for right u-ASCP. All functional variants are depicted in Figure 2.

\section{Functional Variants for Safe u-ASCP}

Seventy-two percent of patients had a functional anatomy safe for right u-ASCP, including a functionally complete $\mathrm{CoW}$ in $27 \%$ of patients $(\mathrm{n}=107)$, isolated right PCoA dysfunction in $10 \%(\mathrm{n}=41)$, isolated left PCoA dysfunction in $13 \%(\mathrm{n}=50)$, and bilateral PCoA dysfunction in $17 \%$ of patients $(n=65)$. A right fetal variant, consisting of aplasia of the right P1 segment, was found in 3\% of patients $(n=11)$. In this variant, the right PCoA supplies the right posterior territory. A combination of left PCoA dysfunction and right fetal variant (ie, aplasia of the right P1 segment) was observed in $2 \%(n=7)$.

\section{Functional Variants for Moderately Safe u-ASCP}

In $18 \%$ of patients $(n=70)$, functional variants with moderate risk of cerebral malperfusion during u-ASCP were observed. Two functional variants jeopardizing the left posterior territory were observed: a left fetal variant (ie, aplasia of the left P1 segment) occurred in 3\% of patients $(\mathrm{n}=12)$, a bilateral fetal variant (ie, aplasia of the left and right $\mathrm{P} 1$ segments) was observed in $8 \%$ of patients $(\mathrm{n}=30)$. In both variants, perfusion of the left posterior 
TABLE 1. Prevalence of observed functional variants

\begin{tabular}{lc}
\hline \multicolumn{1}{c}{ Functional variant } & n $(\%)$ \\
\hline Safe group & \\
Complete CoW & $107(27)$ \\
Right PCoA dysfunction & $41(10)$ \\
Left PCoA dysfunction & $50(13)$ \\
Right and left PCoA dysfunction & $65(17)$ \\
Right fetal variant & $11(3)$ \\
Left PCoA dysfunction and right fetal variant & $7(2)$ \\
Total safe group & $281(72)$ \\
Moderately safe group & \\
Left fetal variant & $12(3)$ \\
ACoA dysfunction & $9(2)$ \\
Bilateral fetal variant & $30(8)$ \\
ACoA and right PCoA dysfunction & $7(2)$ \\
ACoA dysfunction and right fetal variant & $6(2)$ \\
Right PCoA dysfunction and left fetal variant & $6(2)$ \\
Total moderately safe group & $70(18)$ \\
Unsafe group & \\
ACoA and left PCoA dysfunction & $6(2)$ \\
ACoA dysfunction and left fetal variant & $0(0)$ \\
ACoA dysfunction and bilateral fetal variant & $5(1)$ \\
ACoA and bilateral PCoA dysfunction & $15(4)$ \\
ACoA and right PCoA dysfunction and left fetal variant & $1(<1)$ \\
ACoA and left PCoA dysfunction and right fetal variant & $1(<1)$ \\
Right VA dysfunction & $12(3)$ \\
Total unsafe group & $40(10)$ \\
\hline CoW, Circle of Willis; PCoA, posterior communicating artery; ACoA, anterior \\
communicating artery; VA, vertebral artery.
\end{tabular}

territory is dependent on collateral flow via the ACoA and subsequently via the left PCoA. Because of this indirect collateral route, the left posterior territory is at higher risk of malperfusion. Also, 2 variants with increased risk of malperfusion in the left anterior territory during u-ASCP were observed. First, dysfunction of the ACoA was observed in $2 \%(\mathrm{n}=9)$, dysfunction of the ACoA combined with right fetal variant was observed in $2 \%(n=6)$ and dysfunction of the ACoA combined with dysfunction of the right PCoA was observed in $2 \%(n=7)$. In these situations collateral perfusion of the left anterior territory is necessary via the basilar artery and subsequently left PCoA to the A1 and A2 segments beyond the middle cerebral artery.

\section{Functional Variants Unsafe for u-ASCP}

Functional variants posing high risk of cerebral malperfusion during u-ASCP were observed in $10 \%$ of patients $(\mathrm{n}=40)$. The most prevalent high-risk variant was dysfunctional ACoA combined with bilateral PCoA dysfunction, occurring in $4 \%$ of patients $(n=15)$. In this variant, no CoW-collaterals to the left anterior and left middle territory exist leaving protection of this territory during right u-ASCP to extracranial and leptomeningeal collateral flow and hypothermia. Another high-risk variant is right vertebral artery dysfunction (prevalence $3 \% ; \mathrm{n}=12$ ), jeopardizing the left middle and - to a lesser extent-left anterior and posterior territories. Dysfunction of the ACoA combined with left PCoA, risking ischemia of the left anterior and left middle territory, occurred in $2 \%$ of patients $(n=6)$, ACoA dysfunction combined with bilateral fetal variant-posing the whole left brain at risk-occurred in $1 \%(n=5)$ of patients.

\section{Unsafe Variants for bi-ASCP}

The use of bi-ASCP does not guarantee perfusion of all cerebral territories during circulatory arrest in all variants. If right vertebral artery is dysfunctional, in combination with either bilateral PCoA dysfunction or in combination with a bilateral fetal variant (prevalence $0.5 \% ; \mathrm{n}=2$ ), the whole posterior territory is isolated from CoW flow during bi-ASCP and thus jeopardized.

\section{DISCUSSION}

In this study, we present a functional analysis of the collateral capacity of the CoW in 391 patients scheduled for aortic arch surgery. On the basis of these data, the risk of ischemic brain damage during right $\mathrm{u}$-ASCP $(28 \%)$ is estimated to be substantially higher than during bi-ASCP $(<1 \%)$. We present an intuitive "traffic light algorithm" aiding the surgical team in translating the preoperative functional TCCD data into a risk estimate of brain malperfusion using u-ASCP instead of bi-ASCP (Video 1).

The optimal method for brain protection during aortic arch surgery remains controversial. Despite all efforts, a considerable number of patients still encounter neurological damage during aortic arch reconstruction. A recent pooled analysis of patients who received ASCP estimated the risk of permanent and transient neurological damage to be still as high as 7\% and 9\%, respectively. ${ }^{7}$ Current options for brain protection are systemic hypothermia alone, or with adjunctive selective brain perfusion. Since Kazui and associates described the technique of bi-ASCP, as many as two-thirds of (European) colleagues ${ }^{8}$ adopted this technique but consensus on the necessity to deliver the perfusate unilaterally or bilaterally is still lacking. ${ }^{1}$ This question can be addressed from different perspectives. Many attempts were made to seek the answer in clinical observational evidence; a recent pooled analysis in respectively 3723 patients receiving bi-ASCP versus 3065 patients receiving u-ASCP${ }^{7}$ and 1894 patients receiving $\mathrm{u}-\mathrm{ASCP}$ versus 3206 patients receiving bi-ASCP, ${ }^{9}$ both showed no difference in the rate of permanent neurological dysfunction. This is in line with a recent propensity-matched analysis suggesting no neurological benefit of bi-ASCP over u-ASCP in elective aortic arch surgery. ${ }^{10}$

An in vivo experimental study in 12 pigs without known CoW anatomy of suggested equal, uniform blood distribution in u-ASCP and bi-ASCP measured using perioperative magnetic resonance perfusion imaging and postoperative 


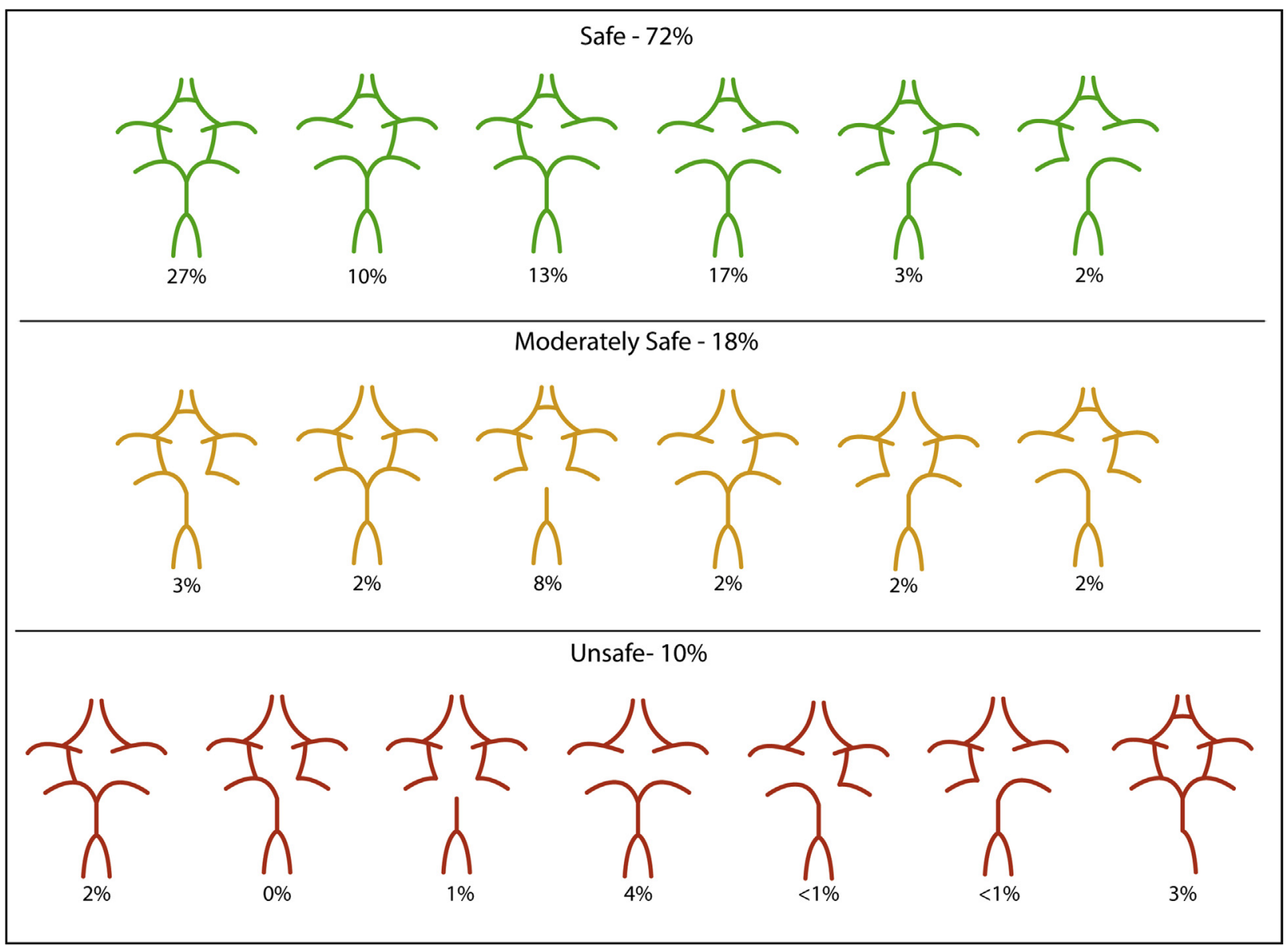

FIGURE 2. Functional variants of the circle of Willis, correlated with safety of unilateral antegrade selective cerebral perfusion.

histopathology of the brain. ${ }^{11}$ In contrary, in vitro simulation of different CoW variants did show unequal blood flow patterns in common anatomical variants. ${ }^{12}$

Morphological studies are also conflicting; dissection studies in human cadavers, and imaging studies of postmortem specimens showed that the $\mathrm{CoW}$ is incomplete in

In case of u-ASCP,

contralateral perfusion depends on collateral pathways, most prominantly the Circle of Willis (CoW)
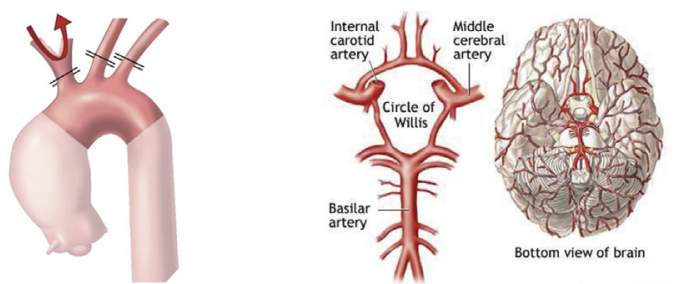

stantonius

VIDEO 1. Video describing the main content of our article. Video available at: https://www.jtcvs.org/article/S0022-5223(19)30030-3/fulltext. almost half of patients and perfusion of the left hemisphere might be compromised in as many as $17 \%$ of patients when considering postmortem vessel diameter as a surrogate marker for ischemia., ${ }^{2,3,13}$ It would be an oversimplification, however, to consider these numbers as approximation of stroke rate if $\mathrm{u}$-ASCP would have been applied in these patients. Apart from the protective effect of hypothermia on the brain, and the collateral capacity of extracranial and leptomeningeal connections, intracranial collaterals are known to have a dynamic nature rather than static. Urbanski and colleagues compared preoperative computed tomography angiography of the CoW with perioperative TCD data. ${ }^{14}$ Although computed tomography angiography suggested incomplete CoW anatomy in $60 \%$ of patients, with critical variants as bilateral absence of PCoA in $13 \%$ and absence of the ACoA as well as bilateral PCoA in $9 \%$, not a single patient appeared to have functional abnormalities as measured perioperatively using TCD during carotid artery occlusion. ${ }^{14}$ Also, magnetic resonance angiography had a weak relationship with functional data in patients. ${ }^{15}$ Our functional assessment of CoW collateral capacity revealed that $72 \%$ of 
patients have a safe functional variant allowing right u-ASCP. In $18 \%$ of patients, the collateral function is uncertain; in $10 \%$ it is considered unsafe because of complete isolation of cerebral territories during right u-ASCP, and thus leaving these territories to the protective effect of hypothermia and extracranial and leptomeningeal connections.

It is conceivable that the reported equal stroke rates after aortic arch surgery using either antegrade cerebral perfusion technique might be confounded by cerebral ischemic damage due to regional contralateral malperfusion using u-ASCP, and inadvertent embolic load by increased manipulation using bi-ASCP, respectively. In our strive to further reduce the incidence of perioperative cerebral damage (permanent and transient) after aortic arch surgery, we propose a tailored approach with full functional assessment preoperatively and subsequently unilateral delivery of the ASCP if safely possible, but bi-ASCP if needed. We propose a "traffic light" algorithm presented in this study as guide for this preoperative decision. If the patient has a "green light" variant, it is appealing to use a right subclavianaxillary approach because this avoids all instrumentation and manipulation of the arch and cerebropetal vessels, avoiding the risks of dislodgment of debris and subsequent embolic stroke. On the basis of our analysis, this would be possible in $70 \%$ of cases. In the unlikely case of perioperative signs of left-sided hypoperfusion on the basis of perioperative TCD flow measurement, bilateral radial artery pressure monitoring, electroencephalography, and near infrared spectroscopy, it takes little time to cannulate the left common carotid ostium additionally. If preoperative functional assessment shows unsafe (amber or red light) variants, we propose to optimally protect the patient using bi-ASCP. We hypothesize that this approach would lead to further decrease of the total neurological damage rate by eliminating unnecessary manipulation and instrumentation of the cerebropetal vessels and its inherent embolic stroke risk when possible, without compromising on intracranial cross-circulation.

An important finding is that Angeloni and coworkers observed an increased rate of mortality in u-ASCP during longer circulatory arrest times. Meta-regression analysis showed increased mortality when u-ASCP was used when circulatory arrest times exceeded 30 minutes. $^{7}$ This association was not observed in bi-ASCP, suggesting that our proposed approach of TCCD-guided u-ASCP should only be used when short circulatory arrest times are anticipated (hemiarch resections only, which account for approximately $70 \%$ of our aortic arch repair population).

\section{CONCLUSIONS}

Complete functional preoperative assessment of the CoW using preoperative TCCD was possible in most aortic arch patients $(61 \%)$. On the basis of these measurements, the risk of malperfusion during right u-ASCP $(28 \%)$ is estimated to be substantially higher than during bi-ASCP $(<1 \%)$. Bi-ASCP is therefore highly preferable over u-ASCP in preventing intraoperative brain damage, especially if the function of the CoW is not known preoperatively. We hypothesize that the risk of neurological damage in aortic arch surgery might be further decreased by using u-ASCP via a right subclavian cannulation if functional anatomy is known to be completely safe, whereas using bi-ASCP in all other variants and thus thereby theoretically reducing the embolic stroke risk of manipulation and instrumentation of the cerebropetal vessels.

\section{Limitation}

We underline that this work describes the functional anatomy (as opposed to morphology, as is seen on imaging) of the CoW as is to be expected during $\mathrm{u}$-ASCP on the basis of TCCD measurements. Further studies are necessary to elucidate how these functional data correlate with the morphological appearance of the $\mathrm{CoW}$ as seen on imaging. Further, we underline that the safety of u-ASCP versus biASCP is on the basis of the theoretical assumptions described in the Methods section. The neurological outcome of our study population was already described by our group, for details we refer to the report of Cefarelli and coworkers. ${ }^{16}$

\section{Conflict of Interest Statement}

Authors have nothing to disclose with regard to commercial support.

\section{References}

1. De Paulis R, Czerny M, Weltert L, Bavaria J, Borger MA, Carrel TP, et al. Current trends in cannulation and neuroprotection during surgery of the aortic arch in Europe. Eur J Cardiothorac Surg. 2015;47:917-23.

2. Papantchev V, Stoinova V, Aleksandrov A, Todorova-Papantcheva D, Hristov S, Petkov D, et al. The role of Willis circle variations during unilateral selective cerebral perfusion: a study of 500 circles. Eur J Cardiothorac Surg. 2013;44:743-53.

3. Merkkola P, Tulla H, Ronkainen A, Soppi V, Oksala A, Koivisto T, et al. Incomplete circle of Willis and right axillary artery perfusion. Ann Thorac Surg. 2006;82:74-9.

4. Dossche KM, Morshuis WJ, Schepens MA, Waanders FG. Bilateral antegrade selective cerebral perfusion during surgery on the proximal thoracic aorta. Eur $J$ Cardiothorac Surg. 2000;17:462-7.

5. Lochner P, Golaszewski S, Caleri F, Ladurner G, Tezzon F, Zuccoli G, et al. Posterior circulation ischemia in patients with fetal-type circle of Willis and hypoplastic vertebrobasilar system. Neurol Sci. 2011;32:1143-6.

6. Hoksbergen AW, Legemate DA, Ubbink DT, de Vos HJ, Jacobs NJ. Influence of the collateral function of the circle of Willis on hemispherical perfusion during carotid occlusion as assessed by transcranial colour-coded duplex ultrasonography. Eur J Vasc Endovasc Surg. 1999;17:486-92.

7. Angeloni E, Melina G, Refice SK, Roscitano A, Capuano F, Comito C, et al. Inilateral versus bilateral antegrade cerebral protection during aortic surgery: an updated meta-analysis. Ann Thorac Surg. 2015;99:2024-31.

8. Kazui T, Inoue N, Yamada O, Komatsu S. Selective cerebral perfusion during operation for aneurysms of the aortic arch: a reassessment. Ann Thorac Surg. 1992;53:109-14

9. Angeloni E, Benedetto U, Takkenberg JJ, Stigliano I, Roscitano A, Melina G, et al. Unilateral versus bilateral antegrade cerebral protection during circulatory arrest in aortic surgery: a meta-analysis of 5100 patients. J Thorac Cardiovasc Surg. 2014;147:60-7

10. Zierer A, Risteski P, El-Sayed Ahmad A, Moritz A, Diegeler A, Urbanski PP. The impact of unilateral versus bilateral antegrade cerebral perfusion on surgical 
outcomes after aortic arch replacement: a propensity-matched analysis. J Thorac Cardiovasc Surg. 2014;147:1212-7; discussion: 1217-8.

11. Ye J, Dai G, Ryner LN, Kozlowski P, Yang L, Summers R, et al. Unilateral antegrade cerebral perfusion through the right axillary artery provides uniform flow distribution to both hemispheres of the brain: a magnetic resonance and histopathological study in pigs. Circulation. 1999;100(19 suppl):II309-15.

12. Zhu G, Yuan Q, Yang J, Yeo J. Experimental study of hemodynamics in the circle of Willis. Biomed Eng Online. 2015;14(suppl 1):S10.

13. Nordon DG, Rodrigues Junior OF. Variations in the brain circulation - the circle of Willis. J Morphol Sci. 2012;29:243-7.

14. Urbanski PP, Lenos A, Blume JC, Ziegler V, Griewing B, Schmitt R, et al Does anatomical completeness of the circle of Willis correlate with sufficient cross-perfusion during unilateral cerebral perfusion? Eur J Cardiothorac Surg. 2008:33:402-8

15. Morita S, Yasaka M, Yasumori K, Oishi Y, Takaseya T, Sonoda H, et al. Transcranial Doppler study to assess intracranial arterial communication before aortic arch operation. Ann Thorac Surg. 2008;86:448-51.

16. Cefarelli M, Murana C, Surace GG, Castrivinci S, Jafrancecso G, Kelder JC, et al. Elective aortic arch repair: factors influencing neurological outcome in 791 patients. Ann Thorac Surg. 2017;104:2016-23.

Key Words: aortic arch surgery, antegrade selective cerebral perfusion, cerebral protection, circle of Willis

Readers who found these articles interesting may also like to read the following papers found in recent and future issues of our sister publications, Seminars in Thoracic and Cardiovascular Surgery and Operative Techniques in Thoracic and Cardiovascular Surgery!

\section{Adult: Aorta}

Optimal Cerebral Protection Strategies in Aortic Surgery. Lou X, Chen EP. Semin Thorac Cardiovasc Surg. 2019 Summer;31(2):146-152.

The Double-Barreled Endografting Technique in a PatientWith Chronic Type B Aortic Dissection. Masada K, Kuratani T, Shimamura K, Sawa Y. Semin Thorac Cardiovasc Surg. 2019 Summer;31(2):183-186.

Commentary: Paving the Right Road? Estrera AL. Semin Thorac Cardiovasc Surg. 2019 Summer;31(2):187.

Use Side Branch of the Aortic Graft to Facilitate Coronary Reconstruction During Complex Aortic Surgery. Cheng YT, Leb JS, Bloom M, et al. Semin Thorac Cardiovasc Surg. 2019 Summer;31(2):201-206.

Commentary: What if “Cabrol” Lost Half of Its Mustache? Dagenais F. Semin Thorac Cardiovasc Surg. 2019 Summer;31(2):207-208 